\title{
E-Learning en la educación continua de profesionales de la salud de establecimientos del tercer nivel de atención en Lima, Perú
}

\section{E-Learning and continuing education in tertiary health facilities in Lima, Peru}

\author{
Pedro Jesús Mendoza-Arana ${ }^{1,2, a}$, Rudi Amalia Loli-Ponce ${ }^{1, b}$, Rocio Salazar-Tello, ${ }^{1, c}$ \\ ${ }^{1}$ Grupo de Investigación SYSTEMIC, Universidad Nacional Mayor de San Marcos. Lima, Perú. \\ ${ }^{2}$ Academia Nacional de Medicina. Lima, Perú. \\ ${ }^{a}$ Médico cirujano, doctor en medicina. ORCID: https://orcid.org/0000-0002-2750-1804 \\ 'bicenciada en enfermeria, doctora en enfermería. ORCID: https://orcid.org/0000-0003-2749-2538 \\ 'Licenciada en obstetricia, ORCID: https://orcid.org/0000-0002-5955-5444
}

An Fac med. 2020;81(3):316-9. / DOI: https://doi.org/10.15381/anales.v81i3.17910

\section{Correspondencia:}

Pedro Mendoza Arana

pmendozaa@unmsm.edu.pe

Recibido: 28 de mayo 2020

Aprobado: 17 de julio 2020

Publicación en línea: 30 de septiembre 2020

Conflictos de interés: Los autores declaran no tener conflictos de interés.

Fuente de financiamiento: Universidad Nacional Mayor de San Marcos. Proyecto A17010641.

Un resumen del presente estudio fue presentado como poster en el $V$ Congreso Intemacional de Educación Médica - CIEM, Lima, 2019.

Citar como: Mendoza-Arana P, LoliPonce R, Salazar-Tello R. E-Learning en la educación continua de profesionales de la salud de establecimientos del tercer nivel de atención en Lima, Perú. An Fac med. 2020;81(3):316-9. DOI: https://doi.org/10.15381/anales. v81i3.17910.

\section{Resumen}

Objetivos. Identificar el grado de utilización del e-learning por los profesionales de la salud y la relación que existe entre los factores organizacionales y los factores individuales del e-learning, en establecimientos de salud públicos y privados de tercer nivel de atención. Métodos. Investigación no experimental, descriptiva, de correlación, con una muestra de 134 profesionales de la salud de dos hospitales públicos y un hospital privado de Lima, Perú. Resultados. Más del $94 \%$ de los encuestados contaban con una computadora ó laptop, celular y acceso a internet. El 74,63\% habia realizado un curso en línea; $79,10 \%$ ha estado presente en video conferencias. Más del $80 \%$ manifestaron satisfacción por los cursos virtuales y desearian que estos se incrementen. Conclusiones. El uso del e-learning es alto, con un promedio de 105 minutos diarios de acceso a contenidos de capacitación, y que sus modalidades principales fueron las videoconferencias y los cursos online; existió una relación directa entre la utilización de recursos de e-learning para la educación profesional continua y los siguientes factores individuales: dominio del idioma inglés, dominio de redes sociales, y acceso a internet. Por otro lado, no se demuestra una relación entre el empleo de recursos de e-learning para la educación profesional continua y los siguientes factores institucionales explorados: políticas de recertificación profesional, políticas de acreditación institucional, e incentivos para la capacitación.

Palabras clave: eLearning; Recursos Humanos; Educación Continua; Educación Médica; Educación en Enfermería; Perú (fuente: DeCS BIREME).

\section{Abstract}

Objective. To identify the relationship between organizational factors and individual factors of e-learning, with the use of it for the purposes of continued education of health professionals, in public and private hospitals. Methods. A non-experimental research has been developed, with a correlational scope, with a sample of 134 health professionals of one private and two public hospitals in Lima, Peru. Results. The use of e-learning for continued education is high (over 70\%), with an average of 105 daily minutes of access to training content, and that its main modalities are video conferences and online courses. Our resuts suggest that invididual factors are more important than organizational factors as determinants of e-learning for continued education purposes. Conclusions. There is a direct relationship between the use of e-learning resources for continuing professional education and the following individual factors: proficiency in the english language, domain of social networks, internet access. On the other hand, we explored posible relationship between the use of e-learning resources for continuing professional education and the following institutional factors: professional recertification policies, institutional accreditation policies, and incentives for continuous education, concluding there is no evidence of such relationship.

Keywords: Education Distance; Workforce; Education Continuing; Education, Medical; Education, Nursing; Peru (source: MeSH NLM). 


\section{INTRODUCCIÓN}

El desarrollo y mantenimiento de las competencias profesionales de los recursos humanos es uno de los elementos cardinales de todo sistema sanitario, al punto que en el modelo de la Organización Mundial de la Salud, se establece como uno de los seis componentes esenciales de todo sistema, el componente de los recursos humanos ${ }^{(1)}$. Este es un proceso complejo, que se inicia con la formación de los profesionales, en general, en entidades universitarias, su licenciamiento, y mecanismos de mantenimiento de su calidad, a través de procesos de renovación periódica de la licencia de ejercicio profesional, proceso que se da en el Perú bajo la forma de la certificación periódica de competencias profesionales ${ }^{(2)}$. De esta manera, el Estado vela por que los profesionales ya en ejercicio, mantengan sus competencias y se asegure de esta manera una atención de calidad a la población (3).

Las actividades, formales o informales, dirigidas a mantener, desarrollar, actualizar, y mejorar sus conocimientos, actitudes y prácticas profesionales, se denominan en genérico "desarrollo profesional continuo", y para el caso específico de los médicos, educación médica continua o EMC ${ }^{(4)}$. Desde la perspectiva de la gestión del personal en las organizaciones, el subgrupo de actividades formales con esta finalidad se denomina "capacitación" (5).

Las investigaciones al respecto han demostrado que, en efecto, el desarrollo profesional continuo es eficaz, y que se relaciona de manera positiva con un mejor desempeño de los médicos y de las enfermeras, así como con mejores resultados para los pacientes ${ }^{(6,7)}$. La efectividad de estos procesos de educación continua depende de factores macro y factores micro ${ }^{(7)}$. En el nivel macro, que en el presente estudio sintetizamos como factores organizacionales, se encuentran aspectos como el entorno social y regulatorio, la organización de los servicios de salud, la cultura en relación con la capacitación, y los requerimientos de acreditación. En el nivel micro, que en el presente estudio sintetizamos como factores individuales, tenemos la disposi- ción de los individuos a involucrarse en actividades de educación continua, y la disponibilidad de los recursos de educación continua.

También hay estudios que demuestran que, desde el punto de vista del diseño educacional, las actividades de mayor eficacia son aquellas que se relacionan con la práctica cotidiana del profesional, lo cual se relaciona con una adecuada evaluación de necesidades de capacitación, y que emplea metodologías interactivas ${ }^{(6,8)}$.

El advenimiento de las computadoras y del Internet, vienen modificando de manera vertiginosa la oferta educativa. En genérico, las formas educativas basadas en computadora se conocen como e-Learning. El e-Learning incluye seis formas básicas ${ }^{(9)}$ : aprendizaje off-line basado en computadoras, aprendizaje on-line basado en computadoras o redes locales (LAN), aprendizaje basado en juegos de computadoras, cursos abiertos masivos on-line (MOOC), modelos de simulación y realidad virtual, y aprendizaje móvil.

El e-learning se propone como una solución a restricciones previamente existentes para la actualización de los profesionales, como el aislamiento geográfico o la falta de tiempo, pues se trata de modelos educativos que pueden ser asincrónicos; es decir, sin que necesariamente coincidan en el momento los docentes y alumnos, y hasta el costo, pues se cuenta ahora con los llamados recursos de educación abierta o REA, uno de cuyos exponentes más conocidos son los cursos abiertos masivos en línea, o cursos MOOC ${ }^{(10)}$. Se ha demostrado también que estos son tan eficaces como los cursos presenciales, para fines de educación médica continua ${ }^{(11)}$, y de educación continua en enfermeros ${ }^{(12,13)}$. El presente estudio busca determinar el grado de utilización del e-Learning en la educación continua en profesionales de la salud de establecimientos de salud de tercer nivel de Lima Metropolitana, y la relación que existe entre los factores organizacionales y los factores individuales para dicha utilización.

\section{MÉTODOS}

\section{Diseño del estudio}

Se realizó un estudio observacional, descriptivo, correlacional.

\section{Población y muestra}

Se obtuvo una muestra aleatoria de profesionales de la salud en dos hospitales públicos y una clínica privada en Lima Metropolitana; el tamaño requerido de la muestra fue de 108 encuestas, recabándose un total de 134 .

\section{Instrumento}

La unidad de análisis fueron profesionales de la salud con un año o más de labor en el establecimiento, a quienes se aplicó una encuesta autoadministrada. Se empleó un cuestionario diseñado y validado por Veloz-Martinez ${ }^{(14)}$, empleado también en nuestro medio por VasquezSilva ${ }^{(15)}$.

\section{Análisis estadístico}

Los datos obtenidos de las encuestas se almacenaron en una base de datos en Excel 2003, y se procesaron en el programa Epi Info ver 7.0 para Windows (Division of Health Informatics \& Surveillance (DHIS), Center for Surveillance, Epidemiology \& Laboratory Services (CSELS). Estados Unidos de América).

\section{Aspectos éticos}

Se solicitó el correspondiente consentimiento informado a los participantes del estudio.

\section{RESULTADOS}

La muestra estuvo compuesta por 134 profesionales, con una edad promedio de 38,6 años. El 47\% fueron médicos; el $38 \%$, enfermeros; el $15 \%$, otros profesionales. El $44 \%$ de los médicos y el $37 \%$ de los enfermeros, estaban recertificados. El 95\% de los profesionales tenían acceso a Internet, ya sea en sus teléfonos celulares o en otros dispositivos, siendo entre estos de mayor frecuencia las computadoras portátiles, con un 94\% (Tabla 1).

Consultados por la autovaloración de su nivel de dominio de herramientas, en una escala de 1 (muy alto) a 5 (muy bajo), este fue de 2,68 para redes sociales, 2,34 para computadora, 2,30 para internet, y 2,23 para correo electrónico.

Los encuestados reportaron un uso promedio de TIC de 4,15 horas diarias 
Tabla 1. Tecnologías de información y comunicación (TIC) disponibles para actividades educativas (en el hogar o en forma personal), en 134 profesionales de la salud de dos hospitales públicos y un hospital privado de Lima, Perú.

\begin{tabular}{lcc}
\hline Computadora de escritorio & 94 & $70,14 \%$ \\
\hline Computadora portátil (Laptop) & 126 & $94,03 \%$ \\
\hline Tablet & 76 & $56,71 \%$ \\
\hline Palm & 15 & $11,19 \%$ \\
\hline Celular con acceso a internet & 127 & $94,77 \%$ \\
\hline Internet en su domicilio & 125 & $93,28 \%$ \\
\hline Internet inalámbrico en sus dispositivos portátiles & 97 & $72,39 \%$ \\
\hline
\end{tabular}

(DE 3,40), de las cuales, un promedio de 105 minutos diarios (DE 83,72) son empleados en forma efectiva para actividades relacionadas con la educación (leer o descargar libros o artículos de revistas de su profesión o especialidad, cursos en línea, investigaciones, entre otras).

En relación con el idioma, los encuestados estimaron que, en una lectura en idioma inglés de cualquier tema, en promedio logra entender claramente el 53\% del material (DE 27,11), cifra que se mantiene en 51\% (DE 27,07) cuando se trata de lecturas científicas. Asimismo, considera que esta falta de dominio de lectura y comprensión en idioma inglés dificulta las búsquedas de información científica en poco o regular, con una media de 1,88 (DE 0,89) de una escala de ( $0=$ nada; 1=poco, 2 =regular, 3=mucho).

El uso real de las TIC para efectos de educación continua y capacitación llega a un $79 \%$, siendo la modalidad principal las videoconferencias, y la menos frecuente los cursos MOOC, con sólo un 14\% (Tabla 2).

Sin embargo, las actividades en general se consideraron que sí gustaron en 111 de los encuestados (82,83\%), fueron contributivas a más conocimiento para 111 encuestados (82,83\%), y 117 $(87,31 \%)$ consideraron que le gustaría que en su formación profesional se im- plementaran y realizaran más actividades educativas virtuales.

Finalmente, 42 encuestados (31,34\%) sienten que el Hospital lo incentiva o apoya para llevar a cabo su actualización o perfeccionamiento profesional, y 17 $(12,7 \%)$ refieren que existe en su hospital algún incentivo o reconocimiento para los profesionales que se actualizan, mientras que $11(8,2 \%)$ refieren que existe alguna sanción o penalidad para los profesionales que no se actualizan.

\section{DISCUSIÓN}

El nivel de utilización del e-Learning para la educación profesional continua en profesionales de la salud en establecimientos de salud de tercer nivel en el ámbito internacional es muy variable. En médicos generales en Escocia, se ha reportado un nivel de utilización superior al $60 \%{ }^{(16)}$, y de $50 \%$ en médicos de un hospital en Lima, siendo la población encuestada, de médicos residentes, es decir, en proceso de formación ${ }^{(15)}$. El presente estudio identificó un 79\% de uso en personal ya especializado, que es la característica del personal de un establecimiento de tercer nivel de atención, y en ejercicio. En tal sentido, podríamos considerar este nivel de uso como alto.

Tabla 2. Uso de TIC para educación continua y capacitación, en 134 profesionales de la salud de dos hospitales públicos y un hospital privado de Lima, Perú.

\begin{tabular}{l|cc}
\hline Participa en blogs y/o foros de discusión & 48 & $(35,82 \%)$ \\
\hline Ha realizado algún curso(s) en línea & 100 & $(74,63 \%)$ \\
\hline Ha participado en algún curso MOOC & 19 & $(14,18 \%)$ \\
\hline Ha estado presente en videoconferencias & 106 & $(79,10 \%)$ \\
\hline
\end{tabular}

Los factores que afectan la posibilidad de uso de la tecnología para la educación continua, clasifican en factores organizacionales y factores individuales ${ }^{(7)}$. Los factores organizacionales comprenden desde el entorno social y regulatorio, la organización de los servicios de salud, la cultura en relación con la capacitación, y los requerimientos de acreditación. En el presente estudio, la influencia de los elementos organizacionales no es preponderante. Menos de un tercio de los encuestados identificó algún incentivo de su hospital para la educación continua, ni en términos de incentivos reforzadores o positivos, ni en el de sanciones. Sólo un 41\% de los profesionales estaba recertificado, siendo que en la actualidad la recertificación es obligatoria para los profesionales de la salud en el Perú (3), e inclusive es supervisada en su cumplimiento por la Superintendencia Nacional de Salud - SUSALUD. El único factor organizacional que se podría identificar es que los establecimientos son sedes docentes universitarias, y este podría ser un elemento dinamizador de la educación continua; en especial, a juzgar por la modalidad que es la más frecuente, que son las videoconferencias y los cursos en línea, con una frecuencia de uso superior al $70 \%$, si bien no podemos afirmar plenamente esta posibilidad pues requeriría un estudio comparativo entre hospitales docentes y no docentes.

En el nivel personal, tenemos la disposición de los individuos a involucrarse en actividades de educación continua, y la disponibilidad de los recursos de educación continua. Por el lado de la disponibilidad, esta es muy alta; como se ha expresado, el 93\% de los encuestados tiene internet en su domicilio, y el 97\% acceso a internet en sus teléfonos móviles. Igualmente, la experiencia se percibe como muy satisfactoria para los encuestados, siendo que el 94\% refieren que los cursos on line le gustaron, que fueron contributivas a más conocimiento para el $89 \%$, y $94 \%$ consideran que les gustaría que en su formación profesional se implementaran y realizaran más actividades educativas virtuales. Estos datos son significativos por la tendencia actual al empleo de los teléfonos "inteligentes" o smartphones. La investigación sobre 
su uso en el entorno médico sugiere su utilización creciente tanto para efectos de una mejor comunicación entre miembros del equipo de salud, como para la búsqueda de información médica en el momento de su necesidad (17).

La presente investigación muestra que, en lo referido a tiempo, los encuestados emplearon un promedio de 105 minutos diarios para realizar actividades educativas, leer, descargar libros, realizar investigaciones, tal como lo reporta el estudio realizado por Martínez, quien afirma que con el e-learning el alumno es su propio controlador, hace su propio horario, accede a materiales de acuerdo con sus necesidades y el orden que para él sea más fácil ${ }^{(18)}$.

Pensando en responder a esta disposición de los profesionales, la Facultad de Medicina de la Universidad Nacional Mayor de San Marcos, en el año 2017, creó la Unidad de Telesalud cuyo objetivo fue la realización de proyectos de innovación, investigación y desarrollo en el sector de tecnología y salud. La primera investigación realizada en dicha unidad, tuvo por objetivo evaluar la viabilidad para la capacitación virtual a través de videos tutoriales online, la instalación de un aplicativo móvil Ilamado JOIN, además la posibilidad de enviar consultas en forma de casos clínicos anónimos, lo cual permitió el actual desarrollo de un programa de telementoria para médicos del Servicio Rural y Urbano Marginal.

Por lo expuesto, se concluye que el empleo de e-learning para la educación continua es de nivel alto (superior al 70\%), con un promedio de 105 minutos diarios de acceso a contenidos de capacitación, y que sus modalidades principales fueron las video-conferencias y los cursos online. Los datos nos permiten concluir que los factores individuales fueron de mayor importancia que los factores organizacionales, pues existe una relación directa entre la utilización de recursos de e-learning para la educación profesional continua y los siguientes factores individuales: dominio del idioma inglés, dominio de redes sociales, y acceso a internet. No se demostró relación entre el empleo de recursos de e-learning para la educación profesional continua y los siguientes factores institucionales explorados: políticas de recertificación profesional, política de acreditación institucional, incentivos para la capacitación.

\section{REFERENCIAS BIBLIOGRÁFICAS}

1. World Health Organization (WHO) [Internet] Everybody's business - strengthening health systems to improve health outcomes: WHO's framework for action. WHO; Geneva: 2007. Disponible en: http://www.who.int/healthsystems/strategy/ everybodys_business.pdf.

2. CONEAU. Seminario Internacional "Certificación Profesional por Competencias". Lima: CONEAUSINEACE, 2010.

3. CONEAU. La Certificación Profesional en el Perú Lima: CONEAU-SINEASE, 2012

4. Giri K, Frankel N, Tulenko K, Puckett A, Bailey R, Ross $\mathrm{H}$. Keeping Up to Date: Continuing Professional Development for Health Workers in Developing Countries. Technical Brief $N^{\circ} 6$, CapacityPlus - IntraHealth International, Inc. 2012.

5. Guglielmetti P. Gestión de la Capacitación en las Organizaciones de Salud. Lima: Ministerio de Salud, 1998

6. Linch GFC, Lima AAA, Souza EN, Nauderer TM, Paz AA, Da Costa C. An educational intervention impact on the quality of nursing records. Rev. Latino-Am. Enfermagem. 2017;25: e2938. DOI: http://dx.doi.org/1518-8345.1986.2938

7. Alkhazim MA, Althubaiti A, Al-Ateega H, Alkhwaiter $M$, AINasser MM. Delivering Effective Continuous Medical Education in Saudi Arabia: Some Critical Issues. Health Professions Education. 2015; 1:43-49. DOI: http://dx.doi.org/10.1016/j. hpe.2015.11.002
8. Marinopoulos SS, Dorman T, Ratanawongsa N, Wilson LM, Ashar BH, Magaziner JL, et. al. Effectiveness of Continuing Medical Education. Evidence Report/Technology Assessment No. 149 (Prepared by the Johns Hopkins Evidence-based Practice Center, under Contract No. 290-02-0018.) AHRQ Publication No. 07-E006. Rockville, MD: Agency for Healthcare Research and Quality, 2007.

9. Brusamento S, Bhone Myint Kyaw, Lorainne Tudor Car, Li Li, Azeem Majeed, Josip Car. eLearning interventions for continuous medical education of health care professionals on pediatrics. PROSPERO 2017: CRD42017057793

10. Liyanagunawardena TR, Williams SA. Massive Open Online Courses on Health and Medicine: Review. Journal of Medical Internet Research. 2014; 16(8), e191. DOI: http://doi.org/10.2196/jmir.3439

11. Sanaiey, Nahid Zarif. The Comparative Study of the Effectiveness of Using E-Learning, Blended Learning and Presence Learning in Continuous Medical Education. World Journal of Medical Sciences. 2014; 10(4):488-493, 2014. DOI: 10.5829/ idosi.wjms.2014.10.4.1402

12. Dalhem WA, Saleh N. The impact of eLearning on nurses' professional knowledge and practice in HMC. Canadian Journal on Nursing Informatics. 2014; 9 (3-4).

13. Van de Steeg L, IJkema R, Wagner C, Langelaan $M$. The effect of an e-learning course on nursing staff's knowledge of delirium: a before-and-after study. BMC Medical Education. 2015; 15(12). DOI: http://doi.org/10.1186/s12909-015-0289-2

14. Veloz-Martinez MG, Almanza-Velasco E, UribeRavell JA, Libiend-Díaz González L, QuintanaRomero V, Alanís-López P. Uso de tecnologias en información y comunicación por médicos residentes de ginecologia y obstetricia. Inved Med. 2012;1(4):183-9.

15. Vásquez-Silva L, Ticse R, Alfaro-Carballido $L$, Guerra-Castañon F. Acceso, uso y preferencias de las tecnologias de información y comunicación por médicos de un hospital general del Perú. Rev Peru Med Exp Salud Publica. 2015;32(2):289-93.

16. MacWalter $G$, McKay $J$ y Bowie P. Utilisation of internet resources for continuing professional development: a cross-sectional survey of general practitioners in Scotland. BMC Medical Education. 2016; 16:24. DOI 10.1186/s12909-016-0540-5

17. Alqaryan S, Alkhalifa M, Alharbi M, Alabaishi S, Aldrees T. Smartphones and professionalism: A cross-sectional study on interns and final-year medical students. International Journal of Medical Research \& Health Sciences. 2016, 5(9):198-202

18. Martinez C. La educación a distancia: sus características y necesidad en la educación actual. Educación 2008; 17(33): 7-27. 